\title{
Multi-stage Preparation for the Repair of Complicated Skull Defects
}

\author{
Yoshiaki SAKAMOTO and Eric ARNAUD² \\ ${ }^{1}$ Department of Plastic and Reconstructive Surgery, Keio University \\ School of Medicine, Tokyo, Japan; \\ ${ }^{2}$ Unité de Chirurgie Crânio-Faciale, Hôpital Necker-Enfants Malades, Paris, France
}

\begin{abstract}
Cranioplasty following decompressive craniectomy is highly associated with complications. Methods for avoiding these complications have been well-documented; however, approaching reconstruction through the previous incision, especially if it was on or very near the bone defect, can sometimes lead to wound dehiscence and infection, with exposure of the artificial bone. For such complicated cases, we propose a multi-staged preparation that includes creating a delayed skin flap for the actual skull reconstruction. Flap was elevated in three stages, with 4 week intervals between each stage to allow for adequate blood supply. Cranioplasty using custom-made implants was then performed. Four patients (age range, 10-25 years) were prepared for skull reconstruction using this technique. During follow-up period for 12 months, no complications, including infections, exposure of the artificial bone, or flap necrosis, were observed. Despite the multiple stages required, we consider that our technique makes a significant contribution to the literature because it suggests a technique for cranioplasty following decompressive craniectomy that may avoid many of the complications following such cranioplasty using current methods.
\end{abstract}

Key words: cranial defect, cranial reconstruction, cranioplasty, delayed phenomenon

\section{Introduction}

Decompressive craniectomy is a neurosurgical procedure most commonly used for the treatment of traumatic brain injury. After decompressive craniectomy has ensured patient survival, skull reconstruction is required. However, cranioplasty following decompressive craniectomy is highly associated with complications, including infection and wound breakdown. ${ }^{1)}$

To avoid these complications, the timing of the surgical intervention and use of materials have been well-described. ${ }^{2-5)}$ In our experience, however, approaching reconstruction through the previous incision, especially if it was on or very near the bone defect, can sometimes lead to wound dehiscence and infection, with exposure of the artificial bone. Thus, as plastic surgeons involved in wound healing, we think that evaluating the previous skin incision, proposed skin flap, and location of the bone defect is also important. For such complicated cases, we propose a multi-staged preparation that

Received November 17, 2018; Accepted December 28, 2018

Copyright $@ 2019$ by The Japan Neurosurgical Society This work is licensed under a Creative Commons AttributionNonCommercial-NoDerivatives International License. includes creating a delayed skin flap for the actual skull reconstruction.

\section{Patients and Methods}

A consecutive series of patients who required skull reconstruction and who had a previous scar over the bone defect or close to the edge of the defect were included. The new skin incision was made such that its distance from the bony defect's edge was $>2 \mathrm{~cm}$. The skin was then incised, and the skin flap was elevated in multiple stages.

In the first stage of the operation, the skin incision was made, reaching the galea aponeurotica. No further dissection was performed; rather, it was simply cut and closed. If vascularization of the area between the previous scar and the new skin incision was concerning, the length of the incision was extended separately several times.

In the second stage, which was performed 4 weeks after the full-length incision, the skin flap was dissected above the pericranium and elevated to the edge of the cranial defect. If vascularization of the flap was considered adequate, further dissection was performed about $1 \mathrm{~cm}$ from the defect's edge. On the other hand, if vascularization was considered 
suboptimal or inadequate, the dissection was delayed for four more weeks. During each interval period until next stage, the wound care was only to have a shampoo from postoperative 2 days. The skin external agent such as antibiotic ointment therapy was not used.

As the final stage, skull reconstruction using custom-made artificial bone was performed 4 weeks after the delayed skin flap was prepared, with an approach from the prepared incision.

\section{Results}

This technique was applied to four patients ranging in age from 2 to 25 years (average, 12.8 years). The causes of the skull defects were trauma in three patients and cranioplasty for craniosynostosis in one. All patients required three-step operations to prepare the bipedicled flap. During the follow-up period, no complications, including infections, exposure of the artificial bone, or necrosis of the flap, were observed except in one patient. The delayed flap of this patient was successful, but expansion of the scalp caused by the skull reconstruction caused flap congestion and wound dehiscence.
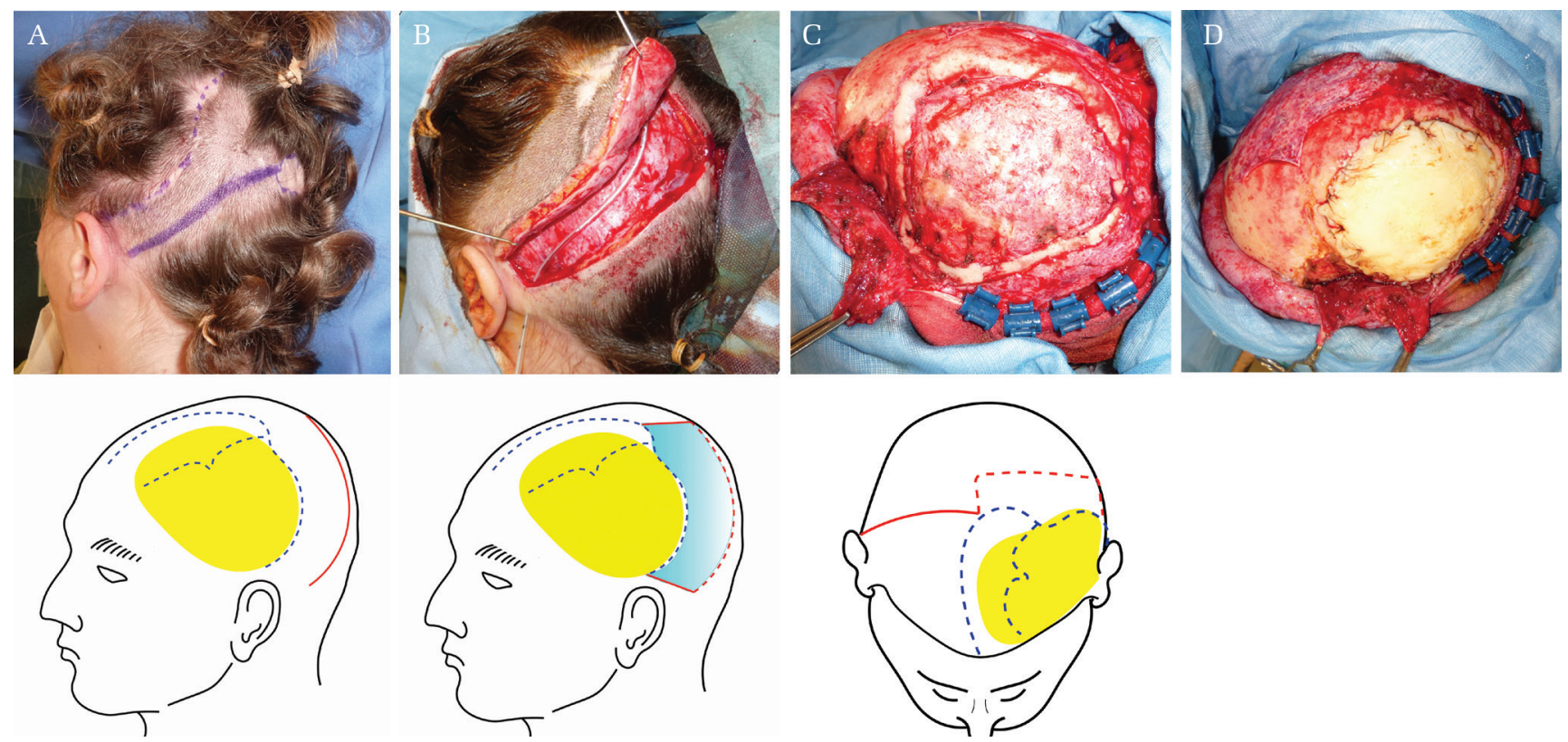

A 25-year-old woman experienced a traumatic skull fracture and underwent an emergency operation. As a result, she had a left frontotemporal bony defect, with a scar just above its rim.

A new skin incision was designed $3 \mathrm{~cm}$ away from the previous incision. Then, the delayed flap was prepared using the two-step operation described above. In the third stage, the artificial bone was implanted (Fig. 1). Postoperatively, the patient experienced no complications and was satisfied with the results.

\section{Discussion}

Wound dehiscence is affected poor focal blood supply and excessive physical stress at wound. ${ }^{6,7)}$ Classically, resection and trimming of previous scar may be effective for focal blood supply. However, the physical stress at wound is going to be increased. To reduce thee physical stress at wound, incision of scaring galea behind the scalp may be essential to mobilize and enlargement of scalp. However, the focal blood supply may be unstable. Soft tissue

Fig. 1 A 25-year-old woman with a left frontotemporal defect. (A) Intraoperative view and schema of the operation's first stage. Note that new skin incision (red line) was designed $3 \mathrm{~cm}$ separating from the previous incision (blue dot line). The previous incision was on the bony edge (yellow area; cranial bone defect). (B) Intraoperative view and schema of the operation's second stage. Note the new skin incision (red line) was added to first stage incision (red dot line). Then, the skin flap was dissected above the pericranium and elevated to the edge of the cranial defect (light blue area). (C) Intraoperative view and schema of the operation's final stage. Note that the bicoronal incision (red line) was performed using the incision prepared by the previous two surgeries (red dot line). This skin incision was separated from the bony edge. (D) Intraoperative view of the operation's final stage. Note that the artificial bone implant was used to fill the defect. 


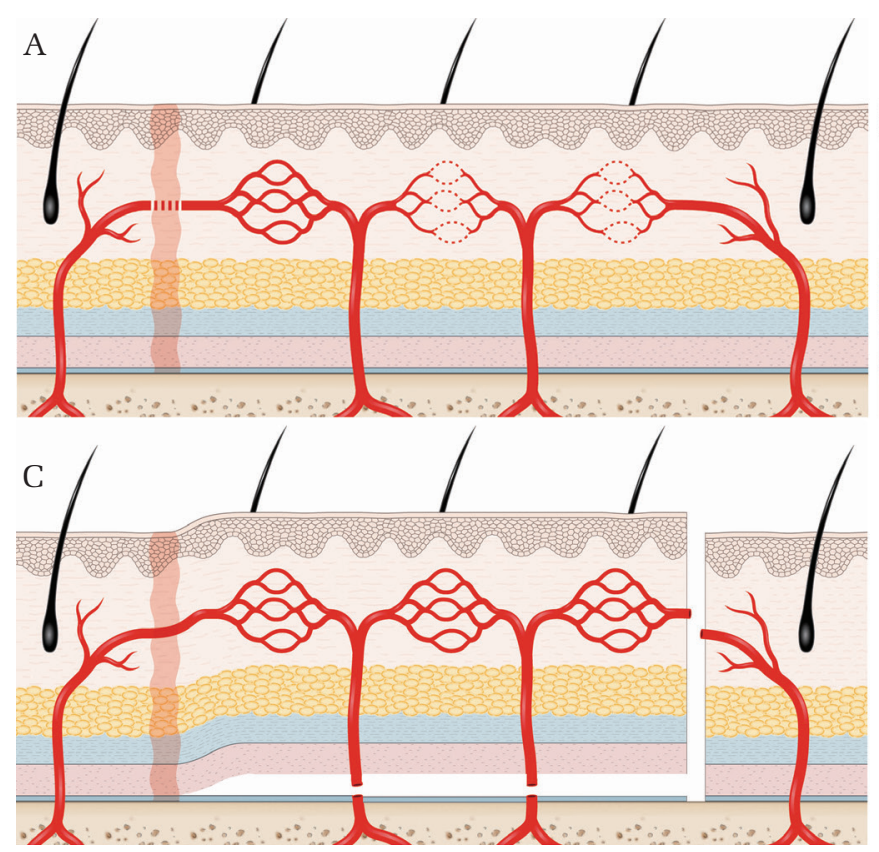

expansion before the cranioplasty can be solved the focal blood supply and physical stress. ${ }^{8,9)}$ However, the complication related to expanders such as infection, exposure, rupture of the expander, and hematoma estimated to $20 \%$ in the literature. ${ }^{10)}$ Also, the patient's discomfort throughout the expansion period, the frequent visits, the esthetic aspect, and the possible pain given by the expander are the disadvantages of expander method. ${ }^{9)}$

In our method, we did not approach reconstruction through the previous scar. Accordingly, the scar remained a "scar" and was not transformed into a "wound"; thus, it remained strong against the physical stress of reconstructive surgery. This method led to a reduced risk of wound dehiscence. However, the area between the new and the previous incisions became a closed area, and low blood supply can provide a risk of necrosis of this area. To improve blood supply to a tissue by creating a surgical wound, we performed the multiple stage preparation known as the delay phenomenon, which is a familiar technique to plastic surgeons.

The physiologic effect of delay is an enlargement of the existing arteries. Blood supply to the incision decreases, and blood supply to other parts compensates for that part. By creating a new incision separate from the previous scar, the blood supply was cut-off, leading to increased blood supply at the previous scar. However, blood supply from the lower floor remains, which is usually formed by reduced-caliber choke arteries that link adjacent cutaneous perforators. When a flap is elevated,

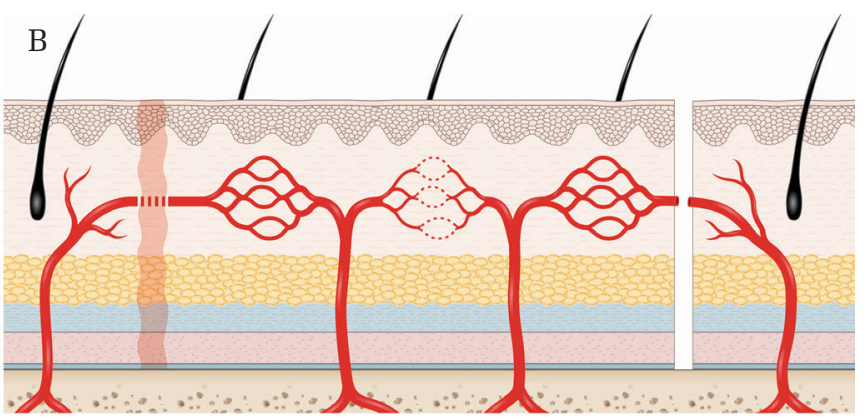

Fig. 2 Schema of the vascularization changes occurring after use of the delay phenomenon. (A) The blood supply was decreased at the previous incision. (B) By creating a new incision separate from the previous scar, the blood supply was cut-off, leading to increased blood supply at the previous scar. (C) When a flap is elevated, the choke vessels, which initially reduce flow from one arterial territory to the next along the flap, enlarge to the caliber of the cutaneous arteries they connect.

these choke vessels, which initially reduced flow from one arterial territory to the next along the flap, enlarge to the caliber of the cutaneous arteries they connect. This phenomenon is a permanent and irreversible process involving multiplication and hypertrophy of the cells in each layer of the vessel wall ${ }^{11,12)}$ (Fig. 2).

The disadvantage of our technique was the number of stages required for preparing a wellvascularized flap. However, each stage was short, lasting approximately 10-15 min. Furthermore, these preparatory operations could be performed under local anesthesia; therefore, we consider that the operative and patient burdens were not high.

In conclusion, our multi-staged preparation for skull reconstruction with a delayed flap is an easy and effective method for treating complicated cranial defects.

\section{Conflicts of Interest Disclosure}

Non-financial interest (such as personal or professional relationships, affiliations, knowledge or beliefs) in the subject matter or materials discussed in this manuscript.

\section{References}

1) Gooch MR, Gin GE, Kenning TJ, German JW: Complications of cranioplasty following decompressive craniectomy: analysis of 62 cases. Neurosurg Focus 26: E9, 2009 
2) Carvi Y, Nievas MN, Höllerhage HG: Early combined cranioplasty and programmable shunt in patients with skull bone defects and CSF-circulation disorders. Neurol Res 28: 139-144, 2006

3) Liang W, Xiaofeng Y, Weiguo L, et al.: Cranioplasty of large cranial defect at an early stage after decompressive craniectomy performed for severe head trauma. J Craniofac Surg 18: 526-532, 2007

4) Lopez J, Zhong SS, Sankey EW, et al.: Time interval reduction for delayed implant-based cranioplasty reconstruction in the setting of previous bone flap osteomyelitis. Plast Reconstr Surg 137: 394e-404e, 2016

5) Manson PN, Crawley WA, Hoopes JE: Frontal cranioplasty: risk factors and choice of cranial vault reconstructive material. Plast Reconstr Surg 77: 888-904, 1986

6) Miller M, Glover D: Wound Management Theory and Practice. London, Emap Healthcare, 1999

7) Reddy S, Khalifian S, Flores JM, et al.: Clinical outcomes in cranioplasty: risk factors and choice of reconstructive material. Plast Reconstr Surg 133: 864-873, 2014

8) Kasper EM, Ridgway EB, Rabie A, Lee BT, Chen C, Lin SJ: Staged scalp soft tissue expansion before delayed allograft cranioplasty: a technical report. Neurosurgery 71: 15-20; discussion 21, 2012

9) Merlino G, Carlucci S: Role of systematic scalp expansion before cranioplasty in patients with craniectomy defects. J Craniomaxillofac Surg 43: 1416-1421, 2015

10) Cunha MS, Nakamoto HA, Herson MR, Faes JC, Gemperli R, Ferreira MC: Tissue expander complications in plastic surgery: a 10-year experience. Rev Hosp Clin Fac Med Sao Paulo 57: 93-97, 2002

11) Cormack G, Lamberry B: The Arterial Anatomy of Skin Flaps. 2nd ed. New York, Churchill Livingstone, 1994

12) Taylor GI, Corlett RJ, Caddy CM, Zelt RG: An anatomic review of the delay phenomenon: II. Clinical applications. Plast Reconstr Surg 89: 408-416; discussion 417-418, 1992

Address reprint requests to: Eric Arnaud, MD, Unité de Chirurgie Crânio-Faciale, Hôpital Necker-Enfants Malades, 149 rue de Sèvres, Paris 75015, France. e-mail: drericarnaud@hotmail.com 